\title{
Detección de un síndrome de Brugada en un reconocimiento médico laboral
}

\section{Detection of a Brugada syndrome in a occupational medical examination}

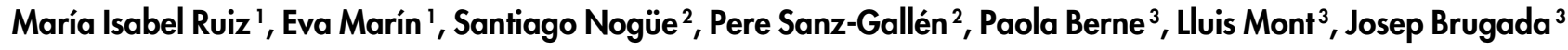 \\ 1. Servicio de Medicina del Trabajo. PREVENGRAF. Terrassa (Barcelona). España. \\ 2. Unidad de Toxicología Clínica. Hospital Clínic. Barcelona. España. \\ 3. Unidad de Arritmias. Servicio de Cardiología. Hospital Clinic. Barcelona. España.
}

Recibido: 15-06-11

Aceptado: 27-07-11

\section{Correspondencia}

Pere Sanz-Gallén

Ronda del General Mitre 39

08017 Barcelona. España.

Tfno: 933203624

Fax: 933203631

e-mail: 17039psg@comb.cat

Resumen

El síndrome de Brugada es una cardiopatía genética y no estructural debida a una alteración primaria de los canales iónicos del miocardio y que se asocia a un riesgo de muerte súbita. Hay tres patrones electrocadiográficos diagnósticos o sugerentes de síndrome de Brugada, que pueden ser identificados en un reconocimiento médico rutinario y que, de confirmarse el diagnóstico, pueden llevar a la necesidad de implantar un desfibrilador automático que puede salvar la vida del paciente. Se presente un caso asintomático diagnosticado en un reconocimiento laboral y se revisa la conducta a seguir ante un síndrome de Brugada.

Med Segur Trab (Internet) 2011; 57 (224) 265-269

Palabras clave: Síndrome de Brugada, Medicina del Trabajo, Medicina Preventiva, Reconocimientos Médicos Laborales.

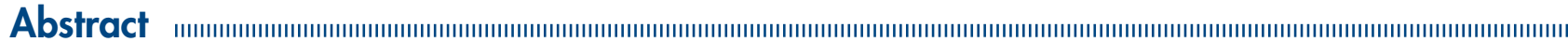

Brugada syndrome is a genetic, non-structural heart disease caused by a primary alteration of the myocardial ion channels and it is associated with increased risk of sudden death. There are three electrocardiographic patterns diagnostic or suggestive of Brugada syndrome which can be identified in routine medical examinations. If the diagnosis is confirmed, implantation of an automatic defibrillator may be life-saving. We report an asymptomatic case of Brugada syndrome diagnosed during an occupational health check and review the steps to be followed after diagnosis of this syndrome.
\end{abstract}

Med Segur Trab (Internet) 2011; 57 (224) 265-269

Key words: Brugada Syndrome, Occupational Medicine, Preventive Medicine, Occupational Medical Examination. 


\section{INTRODUCCIÓN}

El síndrome de Brugada (SB) fue descrito por primera vez en 1992 y se caracteriza por un patrón electrocardiográfico característico en precordiales derechas y la predisposición a presentar arritmias ventriculares y muerte súbita ${ }^{1}$.

El SB es debido a una alteración primaria de los canales iónicos del miocardio pero que no se asocia con una cardiopatía estructural ${ }^{2}$. En los últimos años, se han podido identificar múltiples mutaciones genéticas causales y comprender cuáles son los mecanismos implicados en la aparición del fenotipo característico y los determinantes del pronóstico clínico en los pacientes ${ }^{3}$.

Se han descrito tres patrones ECG distintos para el $\mathrm{SB}^{2}$. El patrón de tipo I se caracteriza por una elevación convexa y descendente del segmento ST $\geq 2 \mathrm{~mm}$ en más de una derivación precordial derecha (V1-V3), seguida de ondas T negativas. En el patrón de tipo II es característica la elevación del segmento $\mathrm{ST} \geq 2 \mathrm{~mm}$ en precordiales derechas seguida de ondas $\mathrm{T}$ positivas $\mathrm{o}$ isobifásicas, lo que confiere al electrocardiograma un aspecto de silla de montar. Finalmente, el patrón de tipo III se define como cualquiera de los dos anteriores si la elevación del segmento ST es $\leq 1 \mathrm{~mm}$. El patrón tipo I es el único que se considera diagnóstico. Los patrones electrocardiográficos tipo II y III son sugestivos pero no diagnósticos de SB, y requieren la realización de un test de ajmalina ó flecainida positivo para la confirmación diagnóstica del síndrome. Los pacientes con síndrome de Brugada permanecen en su mayoría asintomáticos. No obstante, se ha descrito que un $17-42 \%$ de ellos presentan síncope o muerte súbita (MS) como consecuencia de una arritmia ventricular en algún momento de su vida ${ }^{4}$. Ello obliga a considerar como pacientes en riesgo a todos los que presentan un patrón ECG característico, incluso cuando éste aparece de forma aislada.

Se presenta un caso de síndrome de Brugada descubierto de forma casual en un reconocimiento médico laboral.

\section{CASO CLÍNICO}

Un hombre de 35 años, que trabajaba en el mantenimiento de instalaciones eléctricas, acudió al Servicio Médico de Empresa para un reconocimiento rutinario. Se encontraba asintomático. Había sido fumador de 10 cigarrillos/día hasta hacía 8 años. No era consumidor de bebidas alcohólicas, no tenía hábitos tóxicos ni tomaba regularmente ningún medicamento. No refería antecedentes familiares de muerte súbita ni tenía antecedentes personales de síncopes, lipotimias, convulsiones, respiración nocturna agónica o palpitaciones. En el reconocimiento médico no se encontraron alteraciones en el examen físico ni en las exploraciones complementarias rutinarias, con excepción de su ECG basal (Figura 1).

Ante la sospecha de un síndrome de Brugada fue remitido a la Unidad de Arritmias del Hospital Clínic de Barcelona. Tras valoración clínica y electrocardiográfica y después de realizar un test de flecainida, se realizó un diagnóstico de SB. Para completar la estratificación del riesgo, se procedió también a un estudio electrofisiológico el cual mostró intervalos A-H de $128 \mathrm{mseg}$ y $\mathrm{HV}$ de $44 \mathrm{mseg}$. Se realizó un protocolo de estimulación ventricular, sin inducción de arritmias ventriculares sostenidas, por lo cual y en conjunto con los datos clínicos del paciente se concluyó que el SB era de bajo riesgo arrítmico. Se recomendó evitar los fármacos y drogas listados en la Tabla 1 y el tratamiento enérgico de cualquier cuadro febril ya que está demostrado que la fiebre puede desencadenar arritmias ventriculares en pacientes portadores de $\mathrm{SB}^{6}$, citándose también para un estudio genético. Seis meses después, el paciente continúa asintomático. 
Figura 1. ECG de 12 derivaciones que muestra bloqueo A-V de primer grado y patrón tipo 2 de síndrome de Brugada en derivaciones precordiales derechas (supradesnivel del segmento ST $\geq 2 \mathrm{~mm}$ en V1-3, con morfología en silla de montar, seguido de onda T bifásica), sugestivo aunque no diagnóstico de síndrome de Brugada

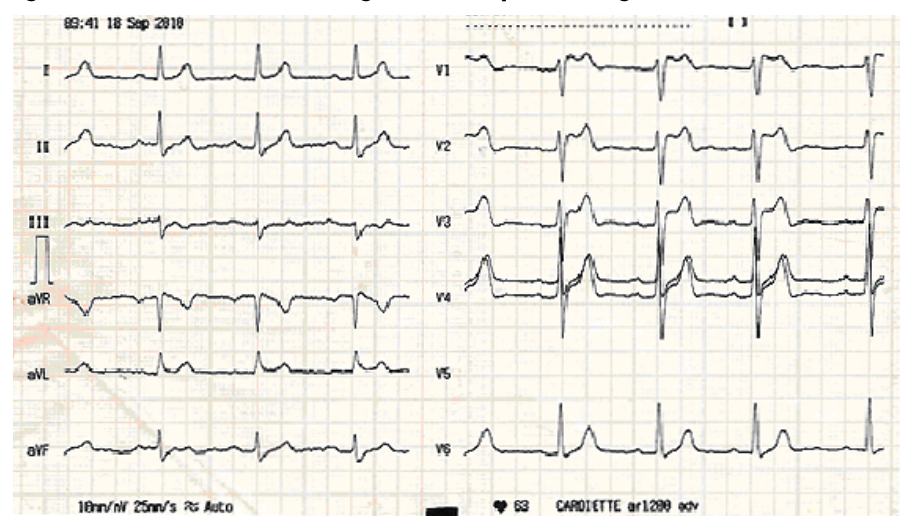

Tabla I. Fármacos y drogas cuyo consumo debería ser evitado en los portadores de un síndrome de Brugado (www.brugadadrugs.org) ${ }^{5}$

\begin{tabular}{|c|c|c|}
\hline \multirow{4}{*}{ Fármacos } & Antiarrítmicos & $\begin{array}{l}\text { Ajamalina } \\
\text { Flecainida } \\
\text { Pilsicainida } \\
\text { Procainamida } \\
\text { Propafenona } \\
\text { Amiodarona } \\
\text { Cibenzolina } \\
\text { Disopiramida } \\
\text { Lidocaína }\end{array}$ \\
\hline & Psicofármacos & $\begin{array}{l}\text { Amitiptilina } \\
\text { Clomipramina } \\
\text { Desipramina } \\
\text { Litio } \\
\text { Loxapina } \\
\text { Nortriptilina } \\
\text { Trifluoperazina } \\
\text { Carbamazepina } \\
\text { Ciamemazina } \\
\text { Dosulepina } \\
\text { Doxepina } \\
\text { Fluoxetina } \\
\text { Fluvoxamina } \\
\text { Imipramina } \\
\text { Maprotilina } \\
\text { Perfenazina } \\
\text { Fenitoína } \\
\text { Tioridazida }\end{array}$ \\
\hline & $\begin{array}{l}\text { Anestésicos/ } \\
\text { Analgésicos }\end{array}$ & $\begin{array}{l}\text { Bupivacaína } \\
\text { Propofol } \\
\text { Ketamina } \\
\text { Tramadol } \\
\end{array}$ \\
\hline & Otras sustancias & $\begin{array}{l}\text { Acetilcolina } \\
\text { Ergonovina } \\
\text { Dimenhidrinato } \\
\text { Edrofonio } \\
\text { Indapamida } \\
\text { Terfenadina/Fexofenadina }\end{array}$ \\
\hline Drogas de abuso & $\begin{array}{l}\text { Alcohol etílico } \\
\text { Cocaína }\end{array}$ & \\
\hline
\end{tabular}




\section{DISCUSIÓN}

El SB se incluye entre las alteraciones cardíacas que se conocen como canalopatías, esto es, enfermedades producidas por alteraciones de los canales iónicos transmembrana que participan en la génesis del potencial de acción celular y cuya consecuencia es la predisposición a la aparición de arritmias. Las canalopatías son enfermedades eléctricas puras y característicamente no se asocian a cardiopatía estructural subyacente ${ }^{2}$.

Se considera que el SB es la causa de un 4-12\% de todas las muertes súbitas y de hasta un $20 \%$ de las muertes súbitas que acontecen en un corazón aparentemente normal. Se calcula que la prevalencia del síndrome de Brugada se sitúa en torno a 5/10.000 habitantes, aunque posiblemente esta cifra subestima la prevalencia real, dado que muchos pacientes pueden presentar formas silentes de la enfermedad.

El SB se transmite característicamente según un patrón de herencia autosómico dominante. No obstante, en una proporción significativa de pacientes, la enfermedad puede ser esporádica, esto es, ausente en otros familiares. Las primeras mutaciones relacionadas con el síndrome de Brugada fueron halladas en 1998 en el gen SCN5A (locus 3p21), que codifica para el canal de sodio cardiaco ${ }^{7}$. Hasta la fecha se han descrito en el mismo gen cerca de 300 mutaciones distintas causantes de SB, cuyo efecto, en todos los casos estudiados, es la reducción de las corrientes transmembrana de sodio (INa), bien sea por una reducción cuantitativa o por una disfunción cualitativa de los canales. A pesar de que el $S C N 5 A$ es el gen que más frecuentemente se asocia a $\mathrm{SB}$, sólo en un $18-30 \%$ de los pacientes es posible identificar una mutación causal en el mismo, lo que indica que la enfermedad es genéticamente heterogénea, lo que se ha confirmado tras el descubrimiento de 7 genes adicionales asociados a $\mathrm{SB}^{8-13}$. Estudios funcionales han demostrado también que aunque no se afecte el canal de sodio, el fenotipo de SB puede explicarse debido a que, de forma similar, se produce un desequilibrio de las corrientes iónicas durante la fase 1 del potencial de acción ${ }^{2,3}$.

El Desfibrilador Automático Implantable (DAI) es el único tratamiento de eficacia realmente demostrada en el síndrome de Brugada ${ }^{14}$. Las indicaciones actuales de DAI corresponden a las recomendaciones propuestas por el II Consenso Internacional publicado en 2005. En general, se implanta a todos los pacientes que ya han tenido síntomas y a las personas asintomáticas en las que el estudio electrofisiológico (EEF) induzca arritmias ventriculares, especialmente si presentan un patrón ECG de tipo I de forma espontánea. En los pacientes asintomáticos, sin historia familiar de muerte súbita y cuyo patrón ECG tipo I sólo se documente tras la administración de fármacos bloqueadores del sodio, se les recomienda evitar una serie de fármacos (www.brugadadrugs.org) ${ }^{5}$, tratamiento agresivo de la fiebre y realizar seguimiento periódico sin necesidad de EEF para su estratificación ${ }^{2}$.

En el ámbito laboral pueden estar presentes riesgos biológicos, físicos y químicos que pueden ocasionar trastornos de la conducción cardiaca. En el caso de un trabajador de mantenimiento de instalaciones eléctricas como el aquí presentado, hay que evitar principalmente las descargas eléctricas que podrían ocasionar arritmias graves, así como la exposición a disolventes orgánicos entre los que destacan los hidrocarburos alifáticos clorados (cloruro de metileno, tricloroetileno, tetracloroetileno, entre otros) por su cardiotoxicidad potencial $^{15}$.

La realización de estudios electrocardiográficos en los exámenes de salud de los trabajadores es una buena herramienta para detectar alteraciones electrocardiográficas asociadas a una muerte súbita como sería el SB.

\section{REFERENCIAS BIBLIOGRÁFICAS}

1. Brugada P, Brugada J. Right bundle branch block, persistent ST segment elevation and sudden cardiac death: a distinct clinical and electrocardiographic syndrome. J Am Coll Cardiol. 1992;20:391-6.

2. Benito B, Brugada J, Brugada R, Brugada P. Brugada Syndrome. Rev Esp Cardiol 2009; 62: 1297-1315.

Detección de un síndrome de Brugada en un reconocimiento médico laboral 
3. Capulzinia L, Brugada P, Brugada J, Brugada R. Arrhythmia and right heart disease: From genetic basis to clinical practice. Rev Esp Cardiol.2010;63:963-83.

4. Brugada J, Brugada R, Antzelevitch C, Towbin J, Nademanee K, Brugada P. Long-term follow-up of individuals with the electrocardiographic pattern of right bundle-branch block and ST-segment elevation in precordial leads V1 to V3. Circulation. 2002;105:73-8.

5. Postema PG, Wolpert C, Amin AS, Boggrefe M, Roden DM. Priori SG, et al. Drugs and Brugada syndrome patients: review of the literature, recommendations, and an up-to-date website (www.brugadadrugs.org). Heart Rhythm 2009;6:1335-1341.

6. Antzelevitch C, Brugada P, Borggrefe M, Brugada J, Brugada R, Corrado D, et al. Brugada Syndrome: Report of the Second Consensus Conference: Endorsed by the Heart Rhythm Society and the European Heart Rhythm Association. Circulation 2005;111:659-670.

7. Chen Q, Kirsch GE, Zhang D, Brugada R, Brugada J, Brugada P, et al. Genetic basis and molecular mechanism for idiopathic ventricular fibrillation. Nature.1998;392:293-6.

8. London B, Michalec M, Mehdi H, Zhu X, Kerchner L, Sanyal S, et al. Mutation in glycerol-3-phosphate dehydrogenase 1-like gene (GPD1-L) decreases cardiac $\mathrm{Na}^{+}$current and causes inherited arrhythmias. Circulation 2007;116:2260-2268.

9. Antzelevitch C, Pollevick GD, Cordeiro JM, Casis O, Saguinetti MC, Aizawa Y, et al. Loss-of-function mutations in the cardiac calcium channel underlie a new clinical entity characterized by ST-segment elevation, short QT intervals, and sudden cardiac death. Circulation 2007;115:442-449.

10. Delpon E, Cordeiro JM, Nunez L, Thomsen PE, Guerchicoff A, Wu Y, et al. Functional effects of KCNE3 mutation and its role in the development of Brugada Syndrome. Circ Arrhythm Electrophysiol 2008;1:209-218.

11. Watanabe H, Koopmann TT, Le Scouarnec S, Yang T, Ingram CR, Schott JJ, et al. Sodium channel beta1 subunit mutations associated with Brugada syndrome and cardiac conduction disease in humans. $\mathrm{J}$ Clin Invest 2008;118:2260-2268.

12. Hu D, Barajas-Martinez H, Burashnikov E, Springer M, Wu Y, Varro A, et al. A mutation in the beta 3 subunit of the cardiac sodium channel associated with Brugada ECG phenotype. Circ Cardiovasc Genet 2009;2:270-278.

13. Medeiros-Domingo A, Tan BH, Crotti L, Tester DJ, Eckhardt L, Cuoretti A, et al. Gain-of-function mutation, S422L, in the KCNJ8-encoded cardiac K ATP channel kir6.1 as a pathogenic substrate for J wave syndromes. Heart Rhythm $2010 ; 7: 1466-1471$.

14. Sacher F, Probst V, Iesaka Y, Jacon P, Laborderie J, Mizon-Gerard F, et al. Outcome after implantation of a cardioverterdefibrillator in patients with Brugada syndrome. Circulation. 2006;114:2317-22.

15. Chamoux A, Malaville P-Y. Pathologie cardiovasculaires professionnelles. EMC ( Elsevier Masson SAS, Paris), Pathologie professionnelle et de l'environnement 16-531-A-10, 2010. 